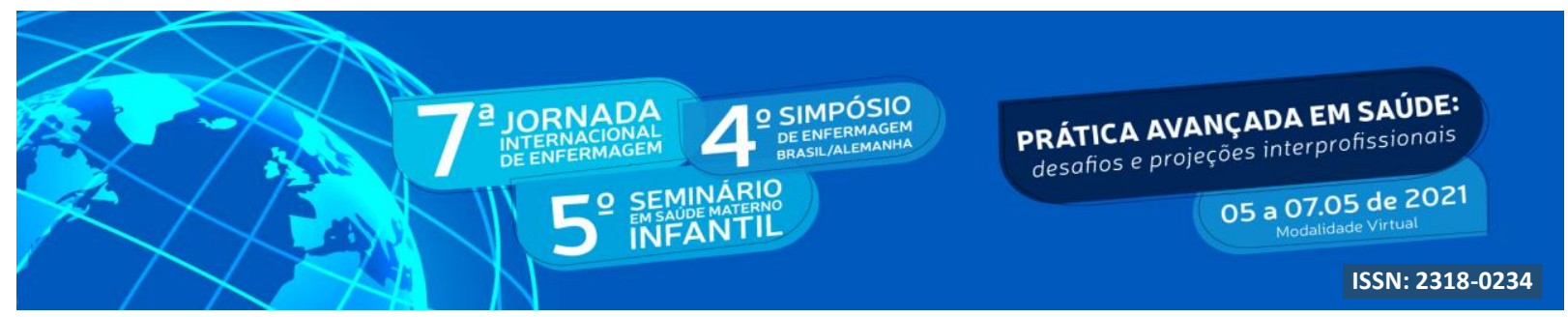

DOI: http://doi.org/10.48195/jie2021-048

\title{
PLANEJAMENTO FAMILIAR E PREVENÇÃO DAS INFECÇÕES SEXUALMENTE TRANSMISSÍVEIS: RODAS DE CONVERSA COM ADOLESCENTES NA ESCOLA ${ }^{1}$
}

\author{
Marcia Dal Bem Cherobini²; Denise Flores de Araujo ${ }^{3}$; Elisiane Possani ${ }^{4}$; Claudia \\ Zamberlan ${ }^{5}$
}

\begin{abstract}
RESUMO
A operacionalização de ações nos espaços educativos, em especial, no ambiente escolar para discutir com os adolescentes aspectos inerentes ao planejamento familiar e prevenção das Infecções Sexualmente Transmissíveis são fundamentais para um viver saudável. Objetiva-se descrever as rodas de conversa desenvolvidas como recurso educativo no processo de ensino-aprendizagem utilizado para adolescentes no cenário escolar. Configura-se como relato de experiência realizado por três mestrandas do Mestrado Profissional em Saúde Materno Infantil da Universidade Franciscana-UFN. A metodologia educativa utilizada com os adolescentes foram rodas de conversa referenciando as temáticas: planejamento familiar e prevenção das Infecções Sexualmente Transmissíveis. Constatou-se que o espaço escolar do referido estudo carece de atividades educativas junto aos adolescentes com a abordagem dessas temáticas que são fundamentais para a saúde sexual e reprodutiva desse grupo populacional.
\end{abstract}

Palavras-chave: Adolescente; Educação em saúde; Escola.

\author{
ABSTRACT \\ FAMILY PLANNING AND SEXUALLY TRANSMITTED INFECTIONS \\ PREVENTION: LEARNING CIRCLES WITH TEENAGERS IN SCHOOL
}

The operationalization of actions in educational spaces, especially in the school environment to discuss with teenagers inherent aspects of Family planning and Sexually Transmitted Infections are fundamental for healthy living. The objective is to describe learning circles developed as an educational resource in the teaching-learning process used for teenagers in the school setting. Such description is an experience report produced by three master's students of the Professional Master's in

\footnotetext{
${ }^{1}$ Relato de experiência.

${ }^{2}$ Enfermeira. Mestranda do Mestrado Profissional em Saúde Materno Infantil- Universidade Franciscana (UFN). Santa Maria, RS. E-mail: marcinhacherobini@gmail.com

${ }^{3}$ Psicóloga. Mestranda do Mestrado Profissional em Saúde Materno Infantil- UFN. Santa Maria, RS E-mail: denisearaujopsi@gmail.com

${ }^{4}$ Enfermeira. Mestranda do Mestrado Profissional em Saúde Materno Infantil- UFN. Santa Maria, RS. E-mail: elisiane.possani@gmail.com

${ }^{5}$ Enfermeira. Docente do Mestrado profissional em Saúde Materno Infantil. UFN. E-mail: czamberlan23@gmail.com
} 


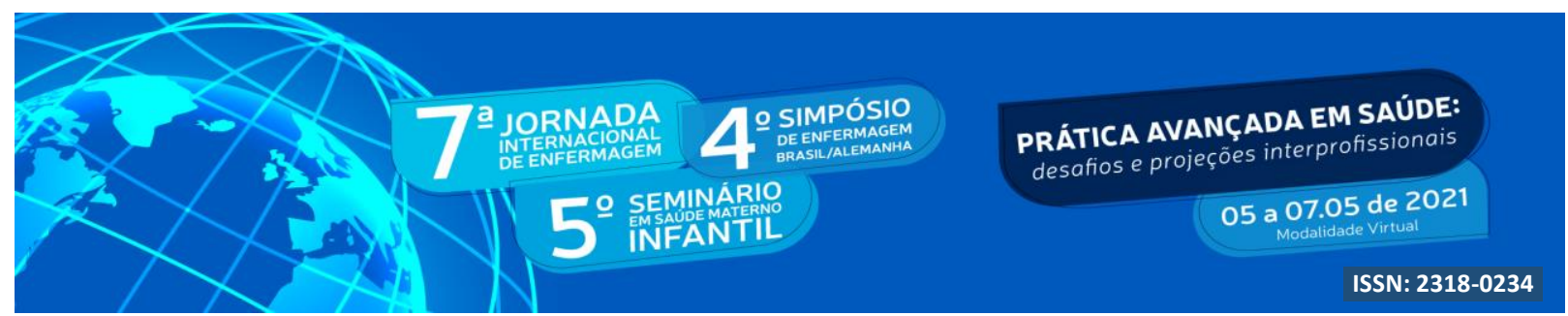

Maternal and Child Health at the Universidade Franciscana (UFN) [Franciscan University]. The educational methodology used with the teenagers was learning circles referring to the themes: family planning and Sexually Transmitted Infections prevention. School space of the referred study was found to lack educational activities alongside teenagers with the approach of these themes that are fundamental for the sexual and reproductive health of this population group.

Key Words: Teenagers; Health education; School.

\section{INTRODUÇÃO}

A adolescência, período compreendido entre 10 aos 19 anos de idade segundo a Organização Mundial da Saúde (OMS), etimologicamente, adulescens significa "aquele que continua a crescer" (ROSA; CARMO-HUERTA, 2020). O despertar para a adolescência possui caráter transversal que envolve não somente modificações fisiológicas, mas também transições, psicológicas e sociais inerentes a essa etapa da vida (ALVES; AGUIAR, 2020). Cabe destacar que muitas dessas transições estão iniciando mais cedo, ou seja, puberdade precoce, casamento em idade mais avançada, globalização e mudança de atitudes e comportamentos sexuais. Tal período pode tornar o adolescente mais vulnerável à práticas sexuais desprotegidas, o que pode ocasionar uma gravidez não planejada e/ou as Infecção Sexualmente Transmissíveis (IST’s) (SBP, 2018).

A Pesquisa Nacional de Saúde do Escolar (PeNSE) evidencia que um terço dos adolescentes iniciam as relações sexuais com menos de 15 anos, boa parte sem proteção e sem método anticoncepcional (NASCER NO BRASIL, 2016). Neste aspecto, destaca-se que os pais, não têm abertura para falar sobre esses temas com seus filhos, sendo que é imprescindível atividades de educação em saúde voltadas para esse grupo populacional proporcionando espaços de diálogo com os adolescentes em relação à saúde sexual e reprodutiva lembrando que os adolescentes divergem quanto aos fatores psicológicos, sociais, culturais e históricos (ROSA; CARMO-HUERTA, 2020).

Dentro dessa perspectiva, em 2019, a convite da equipe diretiva de uma escola estadual em um município do de interior do estado do Rio Grande do Sul (RS), foram realizadas rodas de conversa com os adolescentes referenciando as temáticas prevenção da gravidez na adolescência e IST's. Assim, promover a saúde dos adolescentes, por meio do desenvolvimento de ações educativas demandadas pela própria comunidade escolar, como 


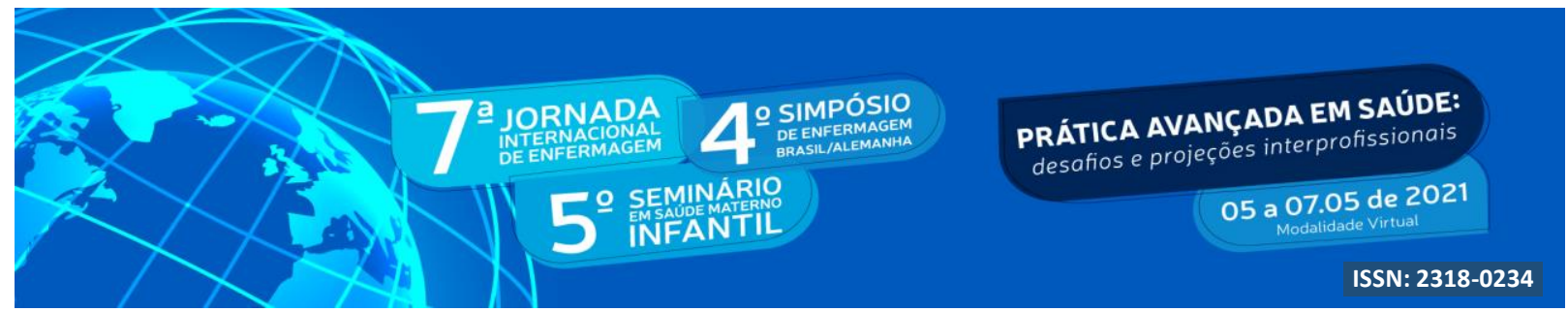

forma de envolver os adolescentes para a promoção de sua saúde, visto que apresentam demandas e necessidades específicas nesse período da vida, além de não aceitarem atitudes impositivas dos educadores e profissionais de saúde.

\section{OBJETIVO}

Descrever as rodas de conversa desenvolvidas como recurso educativo no processo de ensino-aprendizagem utilizado para adolescentes no cenário escolar.

\section{METODOLOGIA}

Trata-se de um relato de experiência referente às rodas de conversa realizadas com adolescentes em uma escola estadual pertencente a um município de interior do estado do RS. Foi desenvolvido por três mestrandas do Mestrado Profissional em Saúde Materno Infantil da Universidade Franciscana (UFN). As atividades foram desenvolvidas nos meses de outubro a novembro de 2019, com duração de aproximadamente 50 minutos para cada turma. A metodologia de intervenção foram rodas de conversa junto aos adolescentes e, as temáticas abordadas foram inerentes ao planejamento familiar e IST's.

\section{RESULTADOS E DISCUSSÃO}

Os profissionais de saúde e educação quando desenvolvem práticas educativas sobre educação para a sexualidade na adolescência precisam estar pautadas em um discurso livre de tabus, pré-conceitos, ou seja, discursos pautados em verdades absolutas e aceitos pela sociedade (RIBEIRO; MAGALHÃES, 2017). Dessa forma, ampliam-se os espaços de discussões com os adolescentes, corroborando com uma maior abertura às discussões e reflexões contemplando assim, todas as formas de opções sexuais que ainda são banalizadas e discriminadas pela sociedade. Nas falas de alguns adolescentes, durante as rodas de conversa, houveram questionamentos sobre o porquê ainda ocorre no ambiente escolar, por parte de alguns educadores, o discurso punitivo para aqueles que fogem de padrões, normas e regras aceitas pela sociedade em relação à identidade de gênero e opção sexual. 


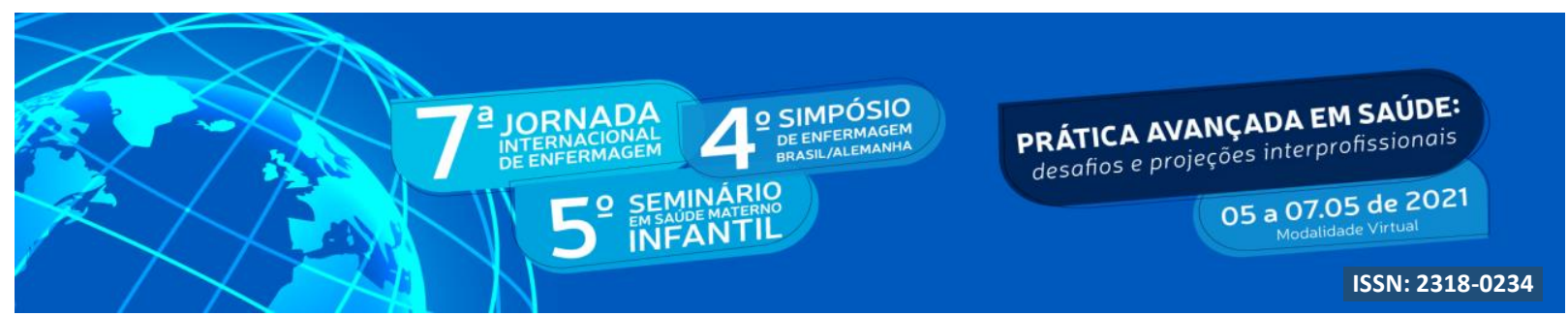

Nesse sentido, percebe-se a relevância dos profissionais de saúde e educação ressignificarem suas concepções e entendimentos sobre sexualidade para assim, ampliarem a abordagem sobre saúde sexual e reprodutiva com os adolescentes transcendendo o conhecimento especifico dessas temáticas e abrindo espaço para discussões mais amplas e abrangentes que problematizem temas que emergem nas atividades educativas. Este fato decorre tendo em vista que a maior parcela dos pais transferem para a escola a abordagem sobre educação para a sexualidade e orientação sobre prevenção da gravidez na adolescência e IST'S, pois esses apresentam dificuldades para sentar com seus filhos e falar abertamente sobre tais temáticas (MULLER, F.C. et al, 2019). Tais aspectos são advindos de questões históricas, culturais, religiosas, políticos ainda atrelados em nossa sociedade conforme Falcoult discute em sua obra intitulada História da Sexualidade (FOUCALT, 1997).

Historicamente, percebe-se nos programas e políticas públicas contempladas nas esferas federais, estaduais e municipais, coordenadas para os profissionais de saúde e educação articularem um cenário de discussões acerca da sexualidade em instituições educacionais referenciando temáticas acerca da sexualidade, planejamento familiar e prevenção das IST's. Nesse contexto, uma dos programas de destaque é o Programa Saúde na Escola (PSE) fundado em 2007, pelo Ministério da Saúde e Educação, que objetiva a articulação entre todos os profissionais envolvidos em desenvolver educação em saúde na escola, inclusive com repasses de verbas para o município que aderir ao programa (BRASIL, 2009).

No entanto, essa articulação no planejamento de uma agenda de atividades educativas na escola ainda é insuficiente na pratica contemporânea, apesar dos esforços relatados pela equipe diretiva da escola do referido estudo, visto que os adolescentes expressaram varias experiências em relação à atividade sexual de risco para gravidez não planejada e IST's. Vislumbrou-se a vulnerabilidade nos relatos das meninas em relação ao uso indiscriminado da pílula de emergência, demostrando assim a percepção de que essa é uma pratica rotineira.

A vivência da sexualidade, na adolescência, em geral manifesta-se através de práticas sexuais desprotegidas, devido à falta de informação, de comunicação entre os responsáveis por esse adolescente, pelas atitudes impensadas e imediatistas, pelo contexto que esta inserido, que são comuns nessa faixa etária e que tornam os adolescentes vulneráveis a 


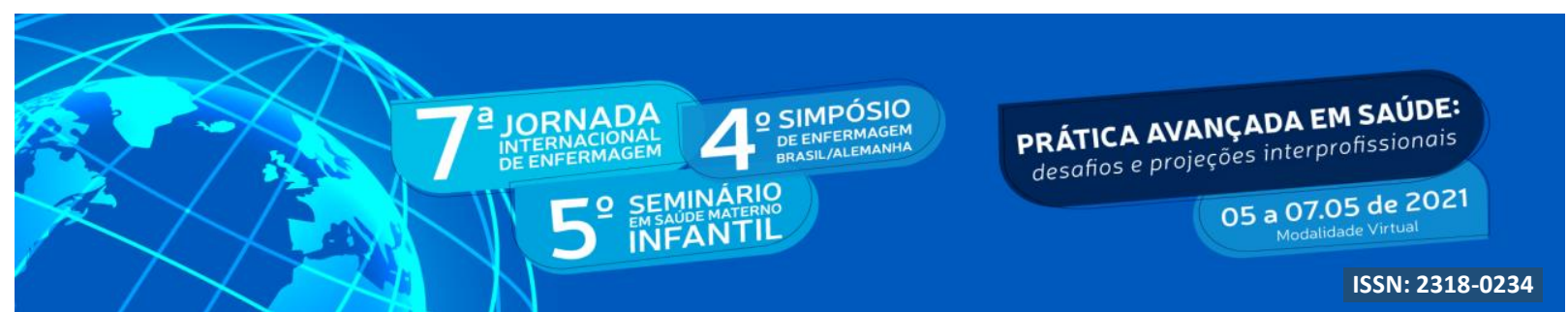

situações de risco, dentre elas destaca-se as IST's e gravidez não planejada (SANTOS et al., 2017; COSTA et al.,2020 ). Nos últimos anos, no Brasil, as IST's, principalmente a AIDS têm aumentado, principalmente entre adolescentes (BRASIL, 2016).

Os profissionais de saúde e educação, como fontes de suporte instrutivo, necessitam habitualmente estimular os adolescentes a procurarem os profissionais de saúde na Unidade Básica de Saúde (UBS) para uma conversa a fim de maiores esclarecimentos sobre uso do preservativo, dos métodos contraceptivos regulares ou de longa duração, da contracepção de emergência e outras orientações que necessitar. Esses são elementos imprescindíveis para a construção da autonomia do adolescente e para o exercício da sexualidade segura com base na perspectiva da sua responsabilidade sobre a sua saúde e bem estar (CABRAL; BRANDÃO, 2020).

\section{CONCLUSÃO}

Evidenciou-se nas rodas de conversa com os adolescentes na escola a necessidade de intensificar estratégias educativas referenciando a temática da prevenção da gravidez na adolescência e IST's, visto que, existe uma carência de informações referentes à esclarecimentos sobre saúde sexual e reprodutiva conforme vislumbrado nas falas dos adolescentes durante as dinâmicas, os quais, demostram em suas falas vivências sexuais de riscos a IST's e gravidez não planejada.

Vislumbra-se a necessidade de uma interlocução entre a comunidade escolar, profissionais de saúde, gestores municipais, família e sociedade para efetivas implementações de ações e serviços voltados aos adolescentes corroborando com seu direto de ser assistido, pois as ações educativas ainda são fragmentadas e insuficientes para a aprendizagem sobre saúde sexual reprodutiva.

Percebeu-se também, a relevância de futuras rodas de conversa/ou outras metodologias que contemplem a ampliação das discussões e temáticas mais abrangentes em relação à orientação sexual e reprodutiva transcendendo discursos direcionados somente para a heteronormatividade, para assim contemplar as diversidades sexuais e de gênero. 


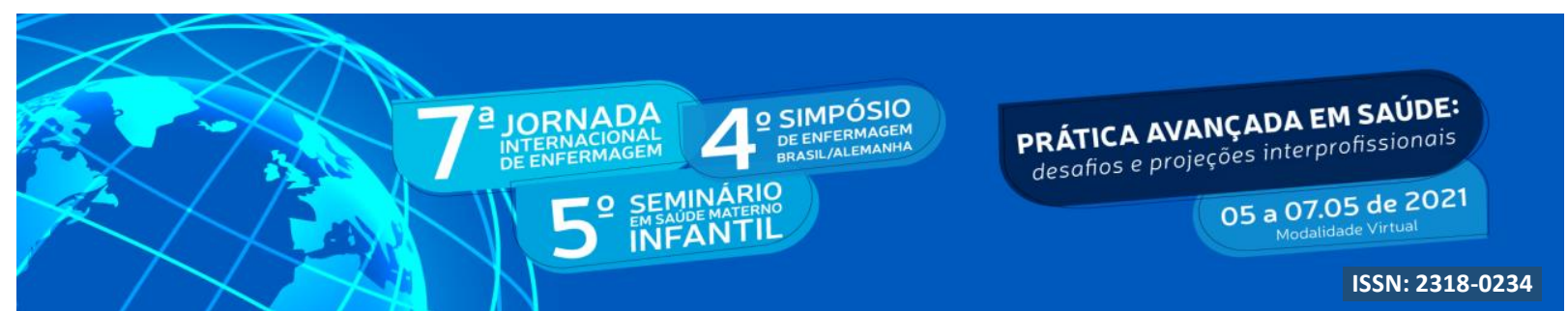

\section{REFERÊNCIAS}

ALVES, L.S; AGUIAR, R.S. Saúde sexual e infecções sexualmente transmissíveis na adolescência. Uma revisão integrativa. Revista Nursing. v. 23, n.263, p.3683-3687, 2020.

BRASIL, Ministério da Educação. Pesquisa nacional de saúde do escolar: 2015 / IBGE, Coordenação de População e Indicadores Sociais. - Rio de Janeiro: IBGE, 2016. 132 p. Convênio: Ministério da Saúde, com apoio do Ministério da Educação Inclui bibliografia e glossário. Disponível em: https://biblioteca.ibge.gov.br/visualizacao/livros/liv97870.pdf. 2016. Acesso em: 02 de març. 2021.

BRASIL. Ministério da Saúde e Ministério da Educação. Programa Saúde na Escola. Brasília: Ministério da saúde, 2009.

BRASIL. Ministério da Saúde. Secretaria de Vigilância em Saúde. Departamento de DST, AIDS e Hepatites virais. Boletim epidemiológico AIDS e DST. Brasília. Ministério da saúde, 2016.

CABRAL, C.S.; BRANDÃO, E. R.. Gravidez na adolescência, iniciação sexual e gênero: perspectivas em disputa. Cad. Saúde Pública. v. 36, n.8,p.1-5, 2020.

COSTA, M.I.F. et al. Adolescentes em situação de pobreza: resiliência e vulnerabilidades às infecções sexualmente transmissíveis. Rev Bras Enferm. v. 73, n.4, p.1-7, 2020.

FOUCALT, Michel. História da Sexualidade I: a vontade de saber. Rio de Janeiro, Edições Graal, 1997.

MULLER, F.C. et al. Sexualidade e prevenção das infecções sexualmente transmissíveis: Empreendedorismo social no ambiente escolar. Evidências empreendedoras na enfermagem: ensino, pesquisa e extensão. Santa Maria: Universidade Franciscana-UFN. p. 286-299, 2019.

RIBEIRO, P. R.C; MAGALHÃES, J.C. Debates contemporâneos sobre Educação para a sexualidade - Rio Grande: ed. Rio Grande: FURG, 2017.

ROSA, M.D.; CARMO-HUERTA, V. O que resta da adolescência: despertar nas fronteiras e nos fronts. Estilos da Clínica. v. 25, n. 1, p. 5-20, 2020.

SANTOS, C. A.N., et al. Análise de Vulnerabilidades em adolescentes escolares. Reon. Facema. V.3, n.1, p.338-344, 2017.

SBP- Sociedade Brasileira de pediatria. Infecções Sexualmente transmissíveis na Adolescência. Guia Prático de Atualização Departamentos Científicos de adolescência e infectologia. n. 6, Agosto de 2018. 